\section{Assessment of visual function in idiopathic intracranial hypertension: a prospective study}

\begin{abstract}
The visual function of 35 patients with a diagnosis of idiopathic intracranial hypertension was assessed prospectively over a 3 year period. In assessing the visual function of cases of idiopathic intracranial hypertension a number of tests were employed including visual field assessment with Humphrey and Goldmann perimeters and documentation of visual acuity and contrast sensitivity. Loss of visual function is the only serious complication and may occur early or late in the course of the condition. An appropriate and sensitive clinical assessment regime is therefore of importance in the outpatient situation. Visual field assessment was documented as the most sensitive to detection of visual loss, with statistically greater sensitivity in comparison with visual acuity and contrast sensitivity testing. Detection of asymptomatic visual loss indicates the necessity for visual monitoring to ensure detection of insidious visual loss. The types of visual field defects noted in this study were typical of anterior optic nerve pathology of raised intracranial pressure and commonly included arcuate defects, nasal steps and global constriction. Visual loss was noted at presentation and during follow-up in up to $87 \%$ of patients using Goldmann perimetry and up to $82 \%$ of patients using Humphrey perimetry. The visual status improved significantly throughout the follow-up period and the final visual outcome was excellent or good in $83 \%$ of patients.
\end{abstract}

Key words Idiopathic intracranial hypertension, Visual function, Visual acuity, Visual field assessment, Contrast sensitivity

Idiopathic intracranial hypertension is a disorder in which there is raised intracranial pressure in the absence of a space-occupying lesion, with a normal cerebrospinal fluid composition and a normal ventricular system in an otherwise healthy patient. The condition was first described in $1893^{1}$ as 'serous meningitis' and terminology has varied considerably over the ensuing years. Terms in current use include idiopathic intracranial hypertension, benign intracranial hypertension and pseudotumour cerebri.

In idiopathic intracranial hypertension, loss of visual function is the only serious complication and may occur early or late in its course. ${ }^{2}$ Visual acuity, peripheral and central fields should all be sequentially recorded. ${ }^{2,3}$ Visual loss is usually insidious and often asymptomatic for long periods of time, and visual disaster can only be anticipated by monitoring the visual fields and acuity. The low incidence of the symptom of visual loss is undoubtedly due to the gradual deterioration of vision that often spares central vision until late in the course of the illness. The frequency of subclinical visual loss underscores the need for thorough ophthalmological examination with perimetry using a disease-specific strategy for patients with idiopathic intracranial

hypertension.

This study compares the methods used for the documentation of visual function in a series of 35 patients and reviews the results of visual field assessment.

\section{Materials and methods}

Thirty-five patients with a diagnosis of idiopathic intracranial hypertension were recruited to this study. There were 30 females and 5 males. The average age at presentation was 29 years (range 11-57 years): 28 years (range 11-57 years) for females and 34 years (range 21-50 years) for males.

The modified Dandy criteria were used in recruitment of patients for this study. ${ }^{4}$ These include: signs and symptoms of increased intracranial pressure, an awake and alert patient, normal neuroimaging studies except for small ventricles or empty sella, documented
F.J. Rowe

N.J. Sarkies

Department of Orthoptics and Ophthalmology

Clinic 3, Box 41

Addenbrooke's NHS Trust Cambridge CB2 2QQ, UK 
increased pressure, ( $>200 \mathrm{mmH}_{2} \mathrm{O}$ in non-obese and $>250 \mathrm{mmH}_{2} \mathrm{O}$ in obese patients ${ }^{5}$ ) with normal cerebrospinal fluid composition, and no other identifiable cause of intracranial hypertension.

The patients were recruited between September 1993 and August 1996 and follow-up ranged from 6 months to 3 years. Patients were seen at 3-month intervals for outpatient assessment, and during inpatient episodes they were also seen on a weekly basis.

Assessment of visual function included documentation of visual acuity using the Snellen chart and visual field assessment with the Humphrey automated and Goldmann manual perimeters. The 24-2 programme was used on Humphrey perimetry, which tested 54 points with a $6^{\circ}$ spaced grid offset from the vertical and horizontal meridians. A modified ArmalyDrance strategy was used on Goldmann perimetry to assess the kinetic boundaries and static points within the central $30^{\circ}$ and temporal sector of the visual field. A grading system was used to classify visual field defects documented with Humphrey and Goldmann perimetry ${ }^{4}$ (Tables 1, 2).
In addition, contrast sensitivity was assessed using the Pelli-Robson chart. Statistical analysis involved use of the SPSS program and the following tests were employed: $t$-test, Wilcoxon matched-pairs sign-rank test, chi-squared test and F-test.

\section{Results}

Visual field assessment: detection of visual field deficit Visual fields were assessed using the Humphrey automated and/or Goldmann manual perimeters. Thirty-five patients (70 eyes) were assessed and each patient was examined at least twice.

At initial examination, a visual field defect was documented in at least one eye in $72 \%$ of patients ( $72 \%$ of eyes) when tested by Goldmann perimetry and in $82 \%$ of patients ( $89 \%$ of eyes) when tested by Humphrey perimetry. During the interim follow-up period, $87 \%$ of visual field tests ( $83 \%$ of eyes) demonstrated a visual field defect by Goldmann perimetry and $80 \%$ of tests $(71 \%$ of eyes) demonstrated a visual field loss defect by Humphrey perimetry. At the

Table 1. Grading system for Goldmann manual perimetry

\begin{tabular}{ll}
\hline Grade 0 & Normal visual field \\
Grade 1 & Minimal visual loss - unlikely to be noticed by the patient
\end{tabular}

Grade $1 \quad$ Minimal visual loss - unlikely to be noticed by the patient

Isopter constriction:

Step defects present that are less than $10^{\circ}$ but greater than $5^{\circ}$ in diameter

Defects not involving fixation:

1. Relative scotomas up to $20^{\circ} \times 20^{\circ}$ in area outside $30^{\circ}$, or up to $10^{\circ}$ in area inside $30^{\circ}$

2 . Blind spot enlargement - encroaches on central $10^{\circ}$

Grade 2 Mild visual field loss - may be noticed by patient and usually compromises function

Isopter constriction:

1. Up to $20^{\circ}$ in area

2. $14 \mathrm{e}$ isopter inside $30^{\circ}$ nasally, $50^{\circ}$ temporally

3. I2e isopter inside $20^{\circ}$

Defects not involving fixation:

1. Relative - less than 1 quadrant in size

2. Absolute - less than $20^{\circ} \times 20^{\circ}$ in area

Defects involving fixation:

VA of $6 / 9$ or better

Grade 3 Moderate visual field loss - nearly always noticed by the patient and which interferes with function Isopter constriction:

1. Greater than $20^{\circ}$ to any isopter but more than $50^{\circ}$ of the field to the V4e target

2. I3e isopter inside the blind spot

3. $12 \mathrm{e}$ isopter inside $10^{\circ}$

Defects not involving fixation:

1. Relative - greater than 1 quadrant but less than 1 hemifield

2. Absolute - greater than $20^{\circ} \times 20^{\circ}$ in diameter but less than 1 quadrant

Defects involving fixation:

VA of $6 / 9$ to $6 / 36$

Grade $4 \quad$ Marked visual field loss

Isopter constriction:

Less than $50^{\circ}$ but greater than $20^{\circ}$ in diameter to V4e

Defects not involving fixation:

1. Relative - 1 hemifield or greater with more than $20^{\circ}$ of field left to V4e

2. Absolute - greater than 1 quadrant with more than $20^{\circ}$ to $\mathrm{V} 4 \mathrm{e}$

Defects involving fixation:

VA of $6 / 36$ to $6 / 60$

Grade $5 \quad$ Blinding visual loss

Isopter constriction:

Less than $20^{\circ}$ to $\mathrm{V} 4 \mathrm{e}$

Defects involving fixation:

Acuity worse than $6 / 60$

From Wall and George. ${ }^{4}$ 


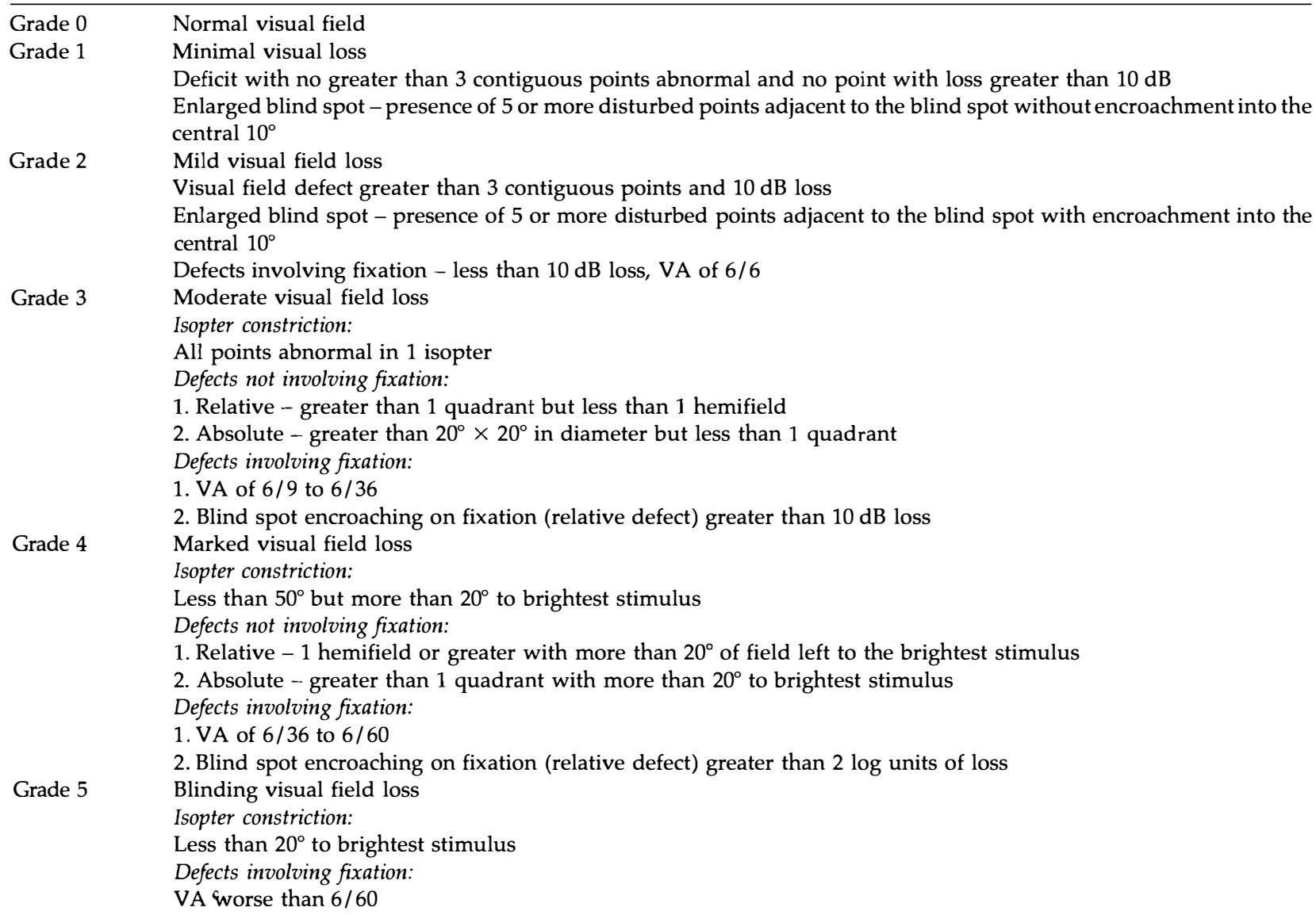

From Wall and George. ${ }^{4}$

last visit, visual field loss was documented in $75 \%$ of patients ( $63 \%$ of eyes) by Goldmann perimetry and $56 \%$ of patients ( $46 \%$ of eyes) by Humphrey perimetry.

Although a considerable number of patients still had visual field loss documented at their last assessment, there was significant improvement in the grade of visual field loss from first to last assessment ( $p=0.0001$ ( $t$-test) and $p=0.0007$ (Wilcoxon test); Figs. 1,2 ). Five patients had required no treatment and retained their good visual function. Thirty patients required medical and/or surgical treatment and the improvement in visual function was most evident in these patients.

The frequency of types of visual field loss was noted. Including initial, interim and final assessments of the 35 patients, a total of 294 tests of visual fields were performed. Of these, $72 \%$ were abnormal and $28 \%$ normal. Most visual field defects corresponded to the presence of papilloedema with pathology of the optic nerve and nerve head. Some functional visual field defects were noted including a monocular temporal hemifield defect documented on two separate occasions and a monocular temporal quadrant defect on another occasion in the same patient. These defects were not found on Goldmann perimetry performed on the same occasion, implying that the defects were functional. All other visual field defects were considered to be real in view of their repeatability and consistency with previous assessments.
All visual fields were graded according to the categories described by Wall and George ${ }^{4}$ and listed in Tables 1 and 2. Grade 0 indicated normal visual fields and grades 1 to 5 indicated visual field defects of increasing severity. The number of eyes falling into each grade was determined (Table 3 ). The most common types of visual field defect were constriction, nasal steps and arcuate defects.

Most visual field defects were minimal or mild (grades 1 or 2), accounting for $67 \%$ of eyes with visual loss. These grades encompassed most types of visual field defects, particularly nasal steps and arcuate defects. Minimal or mild visual field loss is usually not symptom producing and, as such, the patients often appear unaware of the defect(s). These figures are therefore of note. Of the defects in grades 3 to 5 , constriction was most frequently demonstrated in 85 of the 188 eyes in these grades (45\%; $p<0.001$, chi-squared test).

\section{Comparison of tests of visual function}

Visual acuity, contrast sensitivity and visual field assessment results were compared to assess their sensitivity and to determine the most suitable test for detection of visual deficit. On 75 occasions, 150 eyes were assessed using all methods. In 110 eye tests (73\%) an abnormal visual assessment was recorded and in 40 eye tests $(27 \%)$ normal values in all three tests were noted. 


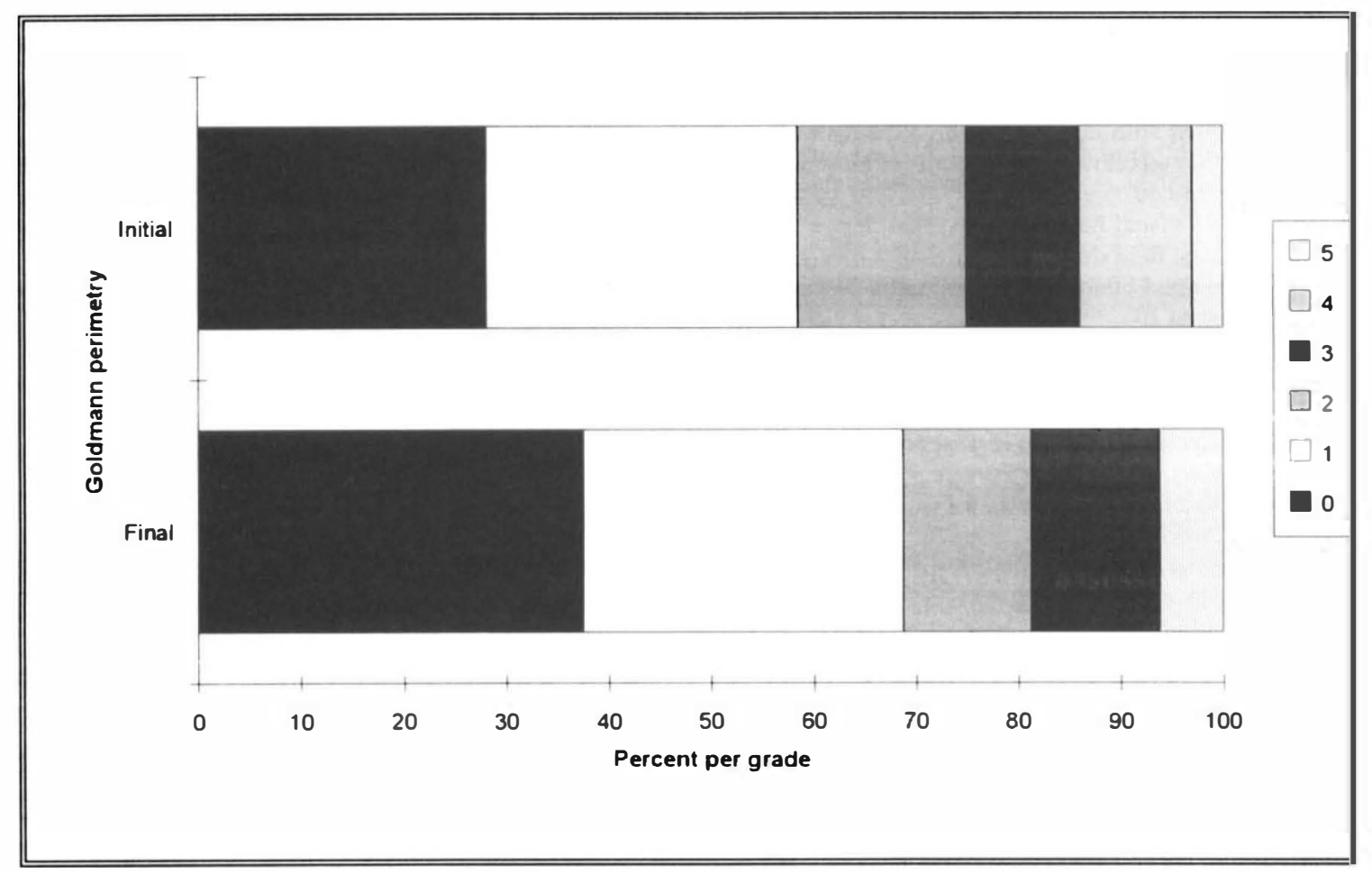

Fig. 1. Improvement of visual field loss from first to last assessment as documented by Goldmann perimetry. Grade 0 indicates a normal visual field and grades 1 to 5 indicate visual field defects of increasing severity. A higher percentage of normal visual fields and grades 1 and 2 are seen at the last assessment.

When comparing the differences seen between the results of contrast sensitivity, visual acuity and visual field assessment in 110 eye tests, abnormal visual field assessment was documented in 103 eye tests (frequency of $94 \%$ ), abnormal contrast sensitivity in 28 eye tests (frequency of $25 \%$ ) and abnormal visual acuity in 36 eye tests (frequency of 33\%). Therefore visual field assessment was more sensitive than visual acuity and contrast sensitivity with a significance of $f=0.0047$ and $f=0.0002$ respectively ( $F$-test). It was apparent that visual field assessment was more sensitive to detection of minimal or mild visual deficit than visual acuity or

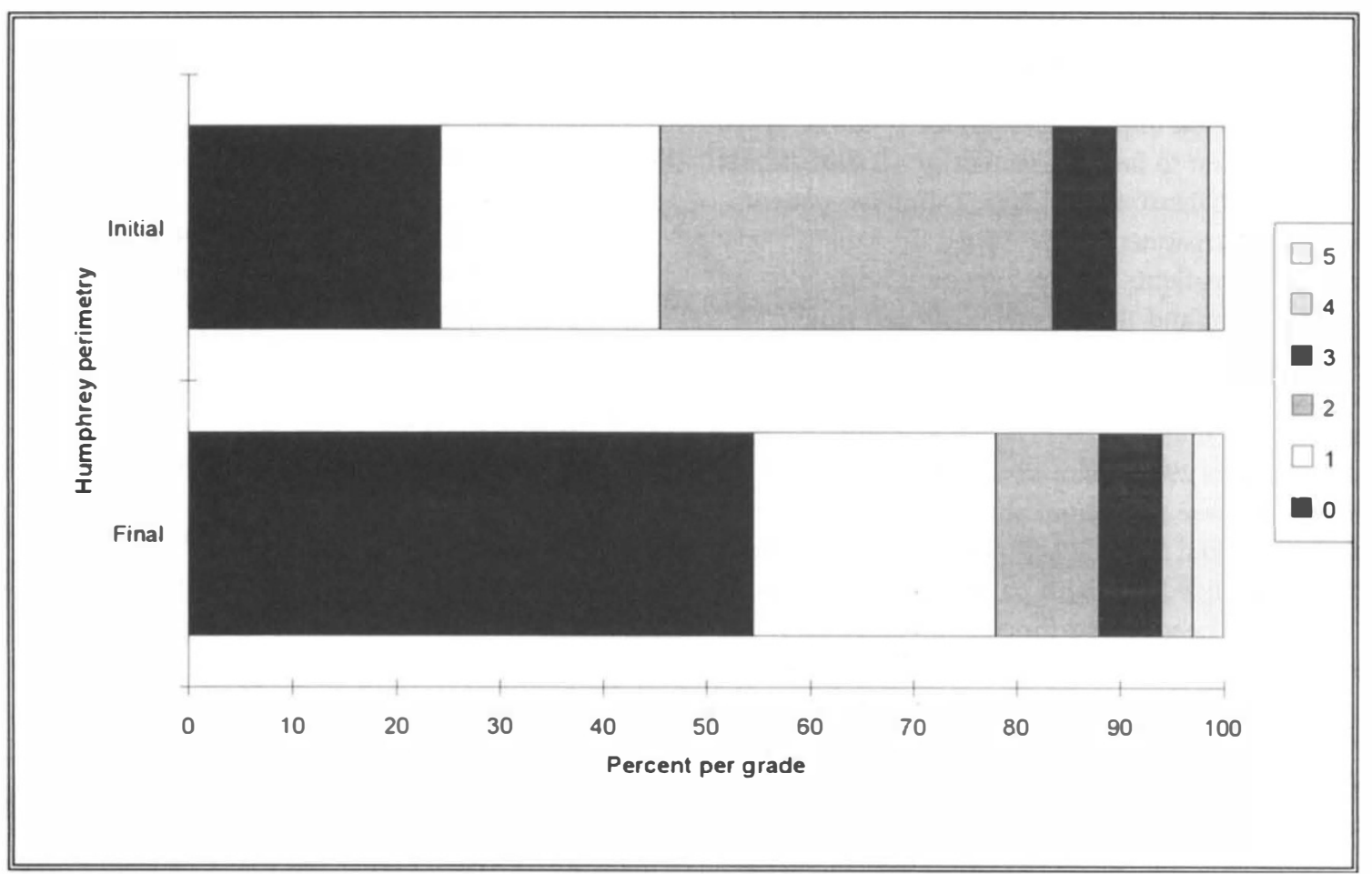

Fig. 2. Improvement of visual field loss from first to last assessment as documented by Humphrey perimetry. Grade 0 indicates a normal visual field and grades 1 to 5 indicate visual field defects of increasing severity. A higher percentage of normal visual fields and grades 1 and 2 are seen at the last assessment. 


\begin{tabular}{|c|c|c|c|c|c|c|}
\hline \multirow[b]{2}{*}{ Visual field defects } & \multirow[b]{2}{*}{ Total } & \multicolumn{5}{|c|}{ Visual field grades } \\
\hline & & 1 & 2 & 3 & 4 & 5 \\
\hline Arcuate defects & 223 & 96 & 79 & 44 & 4 & \\
\hline Nasal steps & 145 & 49 & 59 & 30 & 6 & 1 \\
\hline Global constriction & 116 & 1 & 30 & 37 & 35 & 13 \\
\hline Reduced nasal sensitivity & 33 & 14 & 14 & 4 & 1 & \\
\hline Paracentral scotomas & 31 & 8 & 19 & 4 & & \\
\hline Wedge defects & 10 & 3 & 1 & 6 & & \\
\hline \multirow[t]{2}{*}{ Functional defects } & 3 & & & 3 & & \\
\hline & & $\begin{array}{c}171 \\
(31 \%)\end{array}$ & $\begin{array}{c}202 \\
(36 \%)\end{array}$ & $\begin{array}{l}128 \\
(23 \%)\end{array}$ & $\begin{array}{c}46 \\
(8 \%)\end{array}$ & $\begin{array}{c}14 \\
(2 \%)\end{array}$ \\
\hline
\end{tabular}

Normal visual fields (grade 0) were documented in 166 eye tests.

contrast sensitivity measurements. In addition, visual field assessment was never normal when the visual acuity and contrast sensitivity were both abnormal, further indicating its sensitivity. There was no significant difference in the sensitivity of contrast sensitivity and visual acuity $(f=0.089, F$-test).

\section{Discussion}

\section{Development of visual field defects}

The visual field defects in idiopathic intracranial hypertension are a direct expression of the pathological changes in the optic nerve and optic nerve head. ${ }^{6}$ The mechanism is uncertain but anatomical, physiological and pathological factors concerned with the microcirculation and autoregulation at the disc are all involved. $^{\text {? }}$

The pathogenesis of visual loss is related to the transmission of elevated cerebrospinal fluid pressure down the optic nerve sheath to the optic nerve head. The nerve fibre loss that produces these defects is the result of chronic axoplasmic stasis, infarction of the nerve fibre layer and the constraints of an unyielding scleral canal. Pressure-related compromise of the microvascular supply may be involved. ${ }^{8}$ Loss of visual function may be insidious or sudden in onset as a result of a gradual increase in cerebrospinal fluid pressure around the optic sheath or a sudden increase in optic nerve sheath pressure. A differential sensitivity of the optic nerve head to chronic increased pressure might explain why certain patients have visual loss while others do not. ${ }^{9}$

When undergoing measurement of visual fields, most patients experience a learning curve. ${ }^{10}$ Most patients learn perimetry quickly during the first visual field assessment after the first few stimulus points have been presented and as a result the first test is often accurate. Some patients, however, do not provide an accurate visual field result until the second test. For this reason, there was a minimum of two tests per patient in this study. The learning curve involves learning to respond consistently during the test and, with experience, patients are noted to respond to more dim stimuli and to stimuli presented further away from the central fixation point. ${ }^{11,12}$ Therefore the usual artefact from an initial test is an overall reduction in sensitivity of the visual field. Localised visual field defects that are typically seen with defects relating to papilloedema and optic nerve head pathology are therefore unlikely to be artefacts. Visual artefacts are also due to poor understanding of the test, slow reflexes, concentration failure, positioning problems with edge rim defects and lid artefacts producing superior arcuate defects. The age group of the patients in this study was generally young. They had no other medical problems and had normal reflexes and good attention spans. Nine of the patients required spectacle correction, three for presbyopia. Each patient wore fullframe spectacles that were used during the test. As such, no lens rim defects were encountered. Lid artefacts were also not noted in this group of patients.

The types of field defects in idiopathic intracranial hypertension are typically similar to those found in patients with glaucoma apart from an enlarged blind spot. ${ }^{3,13}$ These nerve fibre bundle defects include inferior nasal defects, ${ }^{6}$ arcuate defects, concentric constrictions, ${ }^{14,15}$ generalised depression of the visual field ${ }^{16}$ and cecocentral scotomas. ${ }^{14,16}$ When bundles of nerve fibres are damaged at the optic disc by papilloedema, the visual field supplied by the fibres loses its sensitivity and the result is a scotoma or a localised depression. Typically the first nerve fibre bundles affected are those entering the upper or lower pole of the optic disc. As a result, paracentral scotomas appear within an arcuate region around fixation, or there is a depression in the nasal field of vision, or both. Less commonly, localised loss of tissue on the nasal side of the optic disc will produce a temporal wedge. ${ }^{17}$

In our study, the most common visual field defects were enlarged blind spots, arcuate defects, nasal steps and global constriction. Other patterns of visual field loss included reduced sensitivity, paracentral scotomas and temporal wedge defects. Most visual field defects were minimal or mild (grades 1 to 2).

Enlarged blind spots are the classic visual field defects in idiopathic intracranial hypertension and are found in virtually all patients with papilloedema. This visual field defect was found in all patients in this study. The enlarged blind spot is rarely noticed by the patient until it encroaches on fixation where there is extension of oedema from the disc into the macula. Where choroidal folds are present, the blind spot may be enormous ${ }^{18}$ and it may continue to be enlarged long after the 
papilloedema has disappeared if the choroidal folds persist. ${ }^{19}$ Paton and Holmes ${ }^{20}$ first described the enlarged blind spot and suggested the cause to be retinal displacement by oedematous optic nerve fibres. Wilbrand and Saenger (quoted by Corbett ${ }^{21}$ ) related blind spot enlargement in papilloedema to lateral retinal displacement and folds in the retina. They also implicated pressure on the circumpapillary retina by the oedematous tissue. Corbett ${ }^{21}$ proposed that an enlarged blind spot was due to peripapillary subretinal fluid collection that elevates the retina from the retinal pigment epithelium and results in local hyperopia.

Nasal visual field loss was a common visual defect. Here, the mechanism is thought to be a combination of mechanical compression and ischaemic damage to axons at the disc. ${ }^{22,23}$ The special susceptibility of the nasal inferior visual field in diseases affecting the optic disc may relate to the regional structural differences in the lamina cribrosa. ${ }^{24}$ Since the greatest density of axons is in the temporal arcuate bundles, it seems plausible that flow stasis and resultant compression would cause defects in these areas more often - thus the greater frequency of nasal as opposed to temporal defects. ${ }^{4}$

Constriction of the visual field is a common consequence of chronic papilloedema. This may begin with small or shallow arcuate scotomas and may progress to a dense, nasal inferior ${ }^{6}$ or superior visual field defect similar to the nasal step seen in glaucoma. Traquair ${ }^{25}$ suggested that the peripheral contraction of the visual field is due to interstitial changes in the optic nerve and is, as a rule, more severe peripherally, with a tendency to spread inward, resulting in a diminution of the blood supply to the nerve fibres in that area.

Constriction of the temporal field, usually seen as a pie-shaped defect pointing to the disc, may occur but is less common and is more typical of an anomalous optic nerve or hypoplastic disc. ${ }^{17}$ These temporal, wedgeshaped defects breaking out horizontally from the blind spot are a distinct perimetric rarity. The defect is due to the absence of nerve fibres in the nasal sector of the retina and optic disc. In the involved eye, the nasal retina appears normal except for the absence of superficial nerve fibre layer striations in the area corresponding to the field defect. This uncommon defect was documented in the visual fields of three patients in this study.

Functional visual field defects were recorded on three occasions from one patient. A monocular temporal hemifield defect was noted on two occasions and a monocular temporal quadrant defect was noted on the third occasion using Humphrey perimetry. Corbett $e t$ al. ${ }^{2}$ noted hysterical visual field defects including tunnel and spiral defects that further complicated the evaluation and management of two patients. Both patients had organic visual field defects present before the hysterical defects appeared and this was particularly difficult in one patient who underwent multiple surgical procedures for what eventually proved to be hysterical visual field loss. In the one patient in this study, the hemifield and quadrant defects were not found when tested on the same visit using Goldmann perimetry, which implicated functional defects. All other visual field defects from assessment on other visits were defects typical of optic nerve head involvement and papilloedema and were considered true defects in view of their repeatability with both methods of perimetry and consistency with previous assessments. However, to clarify further the presence of visual field loss, particularly when considering surgical intervention, the patient underwent electrodiagnostic evaluation that confirmed the presence of a visual deficit.

\section{Comparison of tests of visual function}

Visual field assessment was compared with measurements of visual acuity and contrast sensitivity to determine sensitivity to visual deficit. Visual field assessment was significantly more sensitive than visual acuity and contrast sensitivity for detection of visual deficit and there was no significant difference in the sensitivity of contrast sensitivity and visual acuity to detection of visual deficit.

The level of visual acuity or appearance of the optic nerve head (stage of papilloedema) often do not indicate the state of visual function, and visual acuity usually remains normal in patients with papilloedema except where the condition is of long standing ${ }^{26}$ or where oedema extends into the central $10^{\circ}$ of the visual field. Visual acuity reflects foveal function and does not indicate the state of preservation of either the paracentral or peripheral fields. ${ }^{27}$ We found Snellen visual acuity testing to be insensitive to visual loss found on perimetry. A low incidence of the symptom of visual loss is due probably to the insidious deterioration in vision until late in the course of the condition. ${ }^{4}$

In patients with idiopathic intracranial hypertension, contrast sensitivity testing has been reported to reveal deficits in $50-75 \%$ of eyes tested. ${ }^{4,28,29}$ Low, middle and high spatial frequency loss have been reported and it has been claimed that contrast sensitivity measurement was superior in detecting visual loss in comparison with Snellen acuity tests. ${ }^{4}$ Predominantly low spatial frequency contrast loss has been found, with improvement beginning at days 4-6 following shunting. ${ }^{30}$ These data suggested recovery in slow axoplasmic transport as the mechanism for improvement.

The Pelli-Robson chart was used to measure contrast sensitivity in this study. It is a popular test due to its ease of use and short test time. However, it was significantly less sensitive to detection of visual loss than was visual field assessment. There was no significant difference between contrast sensitivity and visual acuity in determining visual deficit. An abnormal response was often only obtained where a visual field defect of mild or moderate grade or visual acuity of $6 / 12$ or less was documented. More detailed computerised measurement of contrast sensitivity might be more sensitive to detection of visual deficit but is more time consuming and has not yet been proved to be as reliable as visual field assessment. 


\section{Detection of visual loss}

A limited number of studies of visual function in idiopathic intracranial hypertension are prospective and originate from Denmark, USA and Libya. Visual function has been followed with Bjerrum, ${ }^{31,32}$ confrontation, ${ }^{33}$ Goldmann, ${ }^{4,34}$ Octopus $^{34}$ and Humphrey (72 point strategy in central $\left.30^{\circ}\right)^{4}$ perimetry in these studies. Visual field loss was documented in $8-96 \%$ of cases with manual perimetry and in $77.5-92 \%$ of cases with automated perimetry.

To our knowledge, this present study was the first UK study to prospectively study visual function in a consecutive series of patients with idiopathic intracranial hypertension and document the incidence and types of visual defects. Visual loss was detected at presentation or during follow-up in up to $87 \%$ of patients when assessed by Goldmann perimetry and up to $82 \%$ of patients by Humphrey perimetry. The detection of visual field defects is highly dependent on the visual field strategy used. The results of this study were compared with those of Wall and George. ${ }^{4}$ Sixty-seven per cent of the visual field defects in this study were minimal or mild and as a result the patient was unlikely to have been aware of the visual deficit. This finding confirms the necessity for visual monitoring to detect subtle visual loss, particularly that of an insidious nature. Of those patients with moderate or marked visual loss, constriction was the most common visual field defect documented and these patients were generally aware of the presence of visual deficit.

At final assessment, visual field loss was noted in this study in $75 \%$ of patients (63\% of eyes) using Goldmann perimetry and in $56 \%$ of patients ( $46 \%$ of eyes) using Humphrey perimetry. Wall and George ${ }^{4}$ found visual field defects at final assessment in $51 \%$ of eyes using Goldmann perimetry and in $72 \%$ of eyes using Humphrey perimetry. Although visual field defects at last assessment were documented in a large number of patients in our study and in eyes in the study by Wall and George, most of the defects were minimal or mild (grades 1 or 2) and the grades of defects were considerably improved in this study at last assessment in comparison with the visual fields at initial visit. Improvement in visual function was noted particularly in those patients who received medical and/or surgical treatment to lower intracranial pressure.

Despite the detection of visual field defects in a high percentage of patients in this study, the final visual outcome was good. An excellent outcome with normal visual field was noted in $43 \%$ of cases. A good outcome with minimal or mild defects in asymptomatic patients was noted in a further $40 \%$ of cases. Three patients (9\%) had a poor outcome but had presented with visual loss, and optic atrophy was noted on fundus examination. A good outcome could therefore not have been expected for these patients.

\section{Conclusions}

A number of methods were used to assess visual function in patients with idiopathic intracranial hypertension. Visual field assessment using either Humphrey automated or Goldmann manual perimetry was found to be most sensitive in the detection of visual deficit in these patients. Visual field loss was documented at presentation or during follow-up in $87 \%$ of patients assessed by Goldmann perimetry and in $82 \%$ of patients assessed by Humphrey perimetry. The degree of visual field loss was documented as improving significantly from initial to final assessment and throughout the period of follow-up. A variety of visual field defects were documented, most corresponding to the presence of papilloedema; commonly these were arcuate defects, nasal steps, global constriction and paracentral scotoma defects.

Many visual field defects pass unnoticed by the patients, being minimal or mild in degree. It is therefore important to continue visual monitoring throughout the routine follow-up of patients with idiopathic intracranial hypertension in order to detect visual loss and any subtle change on subsequent visits.

If visual field loss can be detected earlier in the course of optic neuropathy and reliably followed for progression, then we may more promptly implement management strategies and determine their effect on arresting and resolving the visual loss in idiopathic intracranial hypertension.

\section{References}

1. Quincke H. Über meningitis serosa. Sammlung Klinische Vortrage (Innere Medezin 23) 1893;67:655-94.

2. Corbett JJ, Savino PJ, Thompson S, Kansu T, Schatz NJ, Orr LS, Hopson D. Visual loss in pseudotumour cerebri. Arch Neurol 1982;39:461-74.

3. Rush JA. Pseudotumour cerebri: clinical profile and visual outcome in 63 patients. Mayo Clin Proc 1980;55:541-6.

4. Wall $\mathrm{M}$, George AE. Idiopathic intracranial hypertension: a prospective study of 50 patients. Brain 1991;114:155-80.

5. Corbett JJ, Mehta MP. Cerebrospinal fluid pressure in normal obese subjects and patients with pseudotumour cerebri. Neurology 1983;33:1386-8.

6. Dersh J, Schlezinger NS. Inferior nasal quadrantanopia in pseudotumour cerebri. Arch Neurol 1959;1:695-9.

7. Orcutt JC, Page NGR, Sanders MD. Factors affecting visual loss in benign intracranial hypertension. Ophthalmology 1984;91:1303-12.

8. Hayreh MS, Hayreh SS. Optic disc oedema in raised intracranial pressure. I. Evolution and resolution. Arch Ophthalmol 1977;95:1237.

9. Gibby WA, Cohen MS, Goldberg HI, Sergott RC. Pseudotumour cerebri: CT findings and correlation with visual loss. AJR 1993;160:143-6.

10. Anderson DR. Automated static perimetry, 1st ed. St Louis: CV Mosby, 1992.

11. Werner EB, Adelson A, Krupin T. Effect of patient experience on the results of automated perimetry in clinically stable glaucoma patients. Ophthalmology 1988;95:764-7.

12. Autzen T, Work K. The effect of learning and age on shortterm fluctuation and mean sensitivity of automated static perimetry [abstract]. Acta Ophthalmol (Copenh) 1990;68:327-30. 
13. Weisberg LA. Benign intracranial hypertension. Medicine 1975;54:197-207.

14. Foley J. Benign forms of intracranial hypertension: toxic and otitic hydrocephalus. Brain 1955;78:1-41.

15. Lysak WR, Svein HJ. Long term follow up on patients with diagnosis of pseudotumour cerebri. J Neurosurg 1966;25:284-7.

16. Smith TJ, Baker RS. Perimetric findings in pseudotumour cerebri using automated techniques. Ophthalmology 1986;93:887-94

17. Buchanan TAS, Hoyt WF. Temporal visual field defects associated with nasal hypoplasia of the disc. $\mathrm{Br} \mathrm{J}$ Ophthalmol 1981;65:636-40

18. Frisen L, Holm M. In: Glaser J, editor. Neuro-ophthalmology, 9th ed. St Louis: CV Mosby, 1977.

19. Corbett JJ, Jacobson DM, Mauer RC, Thompson HS. Enlargement of the blind spot caused by papilloedema. Am J Ophthalmol 1988;105:261-5.

20. Paton L, Holmes G. The pathology of papilloedema: a histological study of 60 eyes. Brain 1911;3:389-432.

21. Corbett JJ. Problems in the diagnosis and treatment of pseudotumour cerebri. Can J Neurol Sci 1983;10:221-9.

22. Minckler D, Tso MOM, Zimmerman RA. A light microscopic, autoradiographic study of axoplasmic transport in optic nerve head during ocular hypotony, increased intraocular pressure, and papilloedema. Am J Ophthalmol 1976;82:741-57.

23. Morse PH, Leveille AS, Antel JP, Burch JV. Bilateral juxtapapillary subretinal neovascularisation associated with pseudotumour cerebri. Am J Ophthalmol 1981;91:312-7.
24. Quigley HA, Addicks EM. Regional differences in the structure of the lamina cribrosa and their relation to glaucomatous optic nerve damage. Arch Ophthalmol 1980;99:137-43.

25. Traquair HM. Introduction to clinical perimetry, 6th ed. St Louis: CV Mosby, 1949.

26. Guidetti B, Giuffre R, Gambacorta D. Follow up study of 100 cases of pseudotumour cerebri. Acta Neurochir 1968;18:259-67.

27. Knight RSG, Fielder AR, Firth JL. Benign intracranial hypertension: visual loss and optic nerve sheath fenestration. J Neurol Neurosurg Psychiatry 1986;49:243-50.

28. Verplanck M, Kaufman DI, Parson T, Yedavally S, Kodinakis D. Electrophysiology versus psychophysics in the detection of visual loss in pseudotumour cerebri. Neurology 1988;38:1789-92.

29. Wall M. Contrast sensitivity testing in pseudotumour cerebri. Ophthalmology 1986;93:4-7.

30. Buncie JR, Tytla ME. Contrast sensitivity in shunted hydrocephalus. Invest Ophthalmol Vis Sci 1989;30:407.

31. Krogsaa B, Sorensen PS, Seedorff HH, Trojaborg W, Gjerris F. Ophthalmologic prognosis in benign intracranial hypertension. Acta Ophthalmol (Copenh) Suppl 1985;63:62-4.

32. Sorensen PS, Krogsaa B, Gjerris F. Clinical course and prognosis of pseudotumour cerebri: a prospective study of 24 patients. Acta Neurol Scand 1988;77:164-77.

33. Radhakrishnan K, Ahlskog JE, Cross SA, Kurland LT, O'Fallon WM. Idiopathic intracranial hypertension (pseudotumour cerebri): descriptive epidemiology in Rochester, Minn., 1976 to 1990. Arch Neurol 1993;50:78-80.

34. Wall M, George DN. Visual loss in pseudotumour cerebri. Arch Neurol 1987;44:170-5. 